\title{
The Case for Using Actigraphy Generated Sleep and Activity Endpoints in Alzheimer's Disease Clinical Trials
}

\author{
M. Mc Carthy, W. Muehlhausen, P. Schüler
}

ICON PLC, South County Business Park, Leopardstown, D18 Ireland

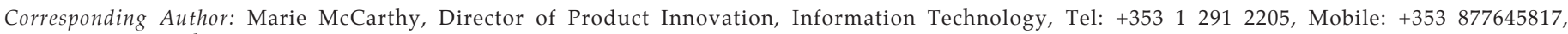
Email: Marie.McCarthy@iconplc.com

J Prev Alz Dis 2016;3(3):173-176

Published online April 22, 2016, http:/ / dx.doi.org/10.14283/jpad.2016.98

\begin{abstract}
More and more people in the industrialised world use wearables and smartphones to monitor their health and fitness. These devices are often used in combination with special apps to monitor and document daily activities and sleep. It would appear to be a logical step to assess the relevance of these devices in drug development trials. In contrast to the consumer devices, the technology used in clinical trials needs to be validated and compliant with the relevant regulations.

Even under these complex requirements, wearables offer a number of new opportunities to objectively capture clinically relevant outcome measures - potentially with lower burden for patients and site staff. As an example, we describe the use in Alzheimer's disease drug development studies. This is an indication where there have been a number of failures, in part due to the difficulties this patient population has in reliably completing existing tools. In addition rater scales add complexity due to inter- and intra-rater variability.
\end{abstract}

Key words: Actigraphy, sleep and activity monitoring, clinical trials, Alzheimer's disease.

\section{Background}

$\mathrm{T}$ The term "wearables" is now ubiquitous and has generated a growing interest in the potential of these devices in drug development trials. Not widely known is that wearables have been used in research to study sleep and activity patterns since the 1970s and gained general acceptance as a diagnostic tool in clinical research into sleep with the publication of the American Academy of Sleep Medicine Practice Parameters in 2007 (1). Wearables are an accepted methodology for tracking activity levels, time spent in moderate and vigorous physical activity (MVPA), and has been used to capture steps and energy expenditure in large community-based studies (2). An analysis of the main clinical trial registries has shown that wearables have been used to generate objective sleep and activity data in drug development trials for over 20 years (3).

The majority of those trials have used the term "actigraphy," a term not well known outside of sleep, activity and circadian rhythm research. Sleep and activity levels have been shown to have clinical significance in the treatment and management of patients suffering from Alzheimer's disease (AD) (4). Traditionally, in-clinic tests such as overnight polysomnography (PSG) and clinical outcomes assessments (COA) have been used to collect sleep and activity levels. However, these tools can be difficult to administer in AD patients. The use of actigraphy derived sleep and activity data gathered in real world non-clinical situations, such as the patient's home or residential care setting, has the potential to offer an alternative means of collecting data that is independent of patient or carer recall (5). In addition, actigraphy addresses artefacts such as first-night effects and other AD patient-related issues that can make it difficult to perform polysomnography in the sleep laboratory (6).

While questions remain around the clinical value of actigraphy and while the logistics of integration into drug development clinical trials can be challenging, there is now a significant body of research supporting the use of actigraphy to generate clinically relevant endpoints. This has been shown by the corpus of 200 articles evaluated for scientific merit in the Ancoli Israel et al review article (6).

\section{What is actigraphy?}

Actigraphy is the study of sleep and activity using gross motor movements generated by accelerometers. These accelerometers are generally wrist worn and can be used for long periods of time; some can be worn for up to 6 months. The activity data generated by these devices is compressed, filtered and analysed using algorithms to generate sleep and activity endpoints. The validity of the technology in the diagnosis of sleep disorders has gained general acceptance with the release of the American Academy of Sleep Medicine (AASM) practice parameters update (1). Accelerometers have also been used in physical activity monitors to measure 
and define physical activity levels. These have been used in large community based studies such as the Centre for Disease Control (CDC), National Health and Nutrition Examination Survey (NHANES) (7) to determine activity levels required for sustained healthy lifestyles.

\section{The relationship between sleep, activity, cognition and Alzheimer's disease}

The greatest utility in AD drug development trials arises from the ability to generate objective measures of sleep and activity levels in non-clinical settings, as shown by Singer et al when they used actigraphy to gather sleep data in private homes and long-term care facilities in a randomized, double blind, placebo-controlled study (8).

The amount of sleep disruption in AD patients usually depends on the stage of their disease. Ju et al (9), in their cross-sectional study, used actigraphy to measure both sleep quality and quantity and showed a differential association between amyloid deposition in the preclinical stage of $\mathrm{AD}$ and sleep quality and quantity.

Changes in circadian rhythms have been regarded as a later consequence of the neurodegenerative disease. However, recent studies have shown that circadian disruption may occur much earlier and may even precede the development of cognitive symptoms (10). More recently, the da Silva review examined sleeps problems in the context of mild cognitive impairment (MCI) and concluded that sleep disturbance is prevalent and predictive of cognitive decline in older people and in those with neurodegenerative disorders (11).

Spira et al (12), in a cross-section study, found that shorter sleep duration and poor sleep quality was associated with greater beta amyloid burden. Virta et al (13), in a follow up study of a Finnish twin cohort, looked at the relationship between beta amyloid and sleep, assessing the sleep characteristics of over 2,000 middle aged twins. The team concluded that while further studies are needed, sleep-related characteristics may emerge as new risk factors for cognitive impairment.

\section{Value of actigraphy in capturing objective sleep data}

PSG is the gold standard for assessing sleep. However, it can be problematic for a cognitively impaired patient to stay in the unknown environment of a sleep lab. The "first night effect" can mean that the data produced are not a true reflection of the sleep patterns in the home environment. Actigraphy has several advantages for patients who are incapable of reliably completing questionnaires or sleep diaries because of cognitive limitations and variability due to inter- and intra-rater variance5, and where laboratory testing is unsuitable (1).

The use of actigraphy in AD drug development trials is supported by significant scientific evidence. A search of ResearchGate revealed over 47 articles with an association between AD and actigraphy. The Camagaros et al (14) review identified 37 individual clinical studies where well-established and validated sleep endpoints were used as either primary or secondary outcome measures. In addition to utilising the well-validated sleep and activity endpoint, there is a case to argue that today we may not be extracting all of the value from this data source. There is potential to identify new and exploratory endpoints with clinical relevance in specific populations using actigraphy data. Exploratory work in other theraputic areas such as pain, autism and COPD has identified endpoints that have value in subtyping patients who show differential responses to therapy (autism)15or who show increased sensivity (pain) (16) or who are at risk of exacerbation (COPD) (17). While it is not feasible to compare one patient population with another, and the current research is limited, activity and sleep patterns can provide signficant insight into patients and their theraputic response with very little patient burden.

\section{Physical activity}

There is considerable interest in investigating the link between physical activity and AD. A search in ResearchGate for "Alzheimer's Disease and Physical Activity" returned over 100 publications. A sedentary lifestyle or the absence of physical activity has been identified by some as a risk factor for AD 4 .

Data from multicentre prospective population cohort studies in Canada18 and Sweden 19 have suggested that physical activity may reduce the risk or delay the onset of dementia or AD.

A number of studies support this association (20), including the Lautenschlager et al (21) randomised controlled trial, which concluded that subjects following a 6-month program of physical activity demonstrated a modest improvement in cognition over an 18-month follow up period. In addition, it has been suggested that physical activity has an impact on improved cognitive function (22) and the progression of AD (23). The link between activity levels and improved cognitive function is not conclusively proven, with some recent review articles failing to identify a link; the most recent Cochrane Review failed to find a causal link among the articles considered (24). However the review did reference 4 other meta-analyses and one systematic review where a positive link was established between physical activity and cognition.

One of the difficulties when comparing studies where physical activity or exercise is an outcome measure is lack of standards. A variety of methodologies and terminology are used to describe and measure physical activity and exercise, such as sedentary bouts, MVPA, steps, metabolic equivalent of task, energy 
expenditure, active energy expenditure, activity counts, cardiorespiratory activity, maximum volume of oxygen and anaerobic exercise. This multitudinous approach makes it very difficult to compare studies and assess the outcome measures.

These studies suggest that activity level in this patient population is a variable that should be measured, monitored and controlled.

A standardisation of approaches would greatly assist future meta-analyses and reviews and provide much needed clarity.

\section{Current clincial trials practice}

While the use of actigraphy in drug development clinical trials is not without precedence, very few $A D$ drug development trials utilise wearables to generate sleep and activity endpoints. In order to assess the usage of "wearables" in drug devolpment trials, an analysis of the FDA's clincial trial registry was carried out. A serach of Clinicaltrials.gov on the 9th of November 2015 generated a list 702 industry-sponsored trials. Of these, 18 trials had "sleep" as an outcome measure, and 170 had "physical activity" as an outcome measure.

From the 170 studies that included physical activity as an outcome measure, trials such as those looking at molecular or brain activity were not included in the analysis. The remaining 151 studies used an array of questionnaires to monitor subject activities. The sole reliance on questionnaires was somewhat surprising due to the difficulties associated with this population. The use of wearables could have provided additional objective data around changes in the patients' activity levels, including changes to sedentary behaviour, time spent in physical activity, and the intensity of the activity or overall activity level. The combination of questionnaires and devices has the potential to authenticate the active periods detailed in the questionnaire and could potentially allow the studies to generate data that are objective and subject to less variability.

The 18 studies with sleep as an outcome measure used a combination of questionnaires, including the Pittsburgh Sleep Quality Index, Epworth Sleepiness Scale and Medical Outcomes Study - Sleep Scale, and laboratory measures such as PSG and EEG to measure changes to sleep pattern and architecture. Of these 18 studies, 4 used actigraphy to record sleep quality and quantity in both phase 2 and 4 studies

\section{Conclusions}

Sleep quality and quantity have clinical relevance in AD. Validated low-patient-burden technology that can objectively measure sleep and activity patterns and is not subject to patient recall bias is available. This technology can be used in the patient's home outside of the clinical setting and has been validated for use in $\mathrm{AD}$ patients. This technology is currently used in clinical and academic research, but it is not used to any great extent in drug development trials, where the utilisation of these devices could help simplify and standardise the collection of objective, clinically relevant endpoints.

The objective measurement of physical activity does not appear to be seen as a clinically relevant endpoint in AD drug development trials. To date, the evaluation of the activity levels of $\mathrm{AD}$ subjects has been restricted to assessment by questionnaires, collating data around specific activities such as time spent at shopping, food preparation, etc. However, absolute changes in activity or sedentary behaviour over the course of a trial, is not currently being assessed. There is still scientific debate around the impact physical activity has on AD, fuelled to some extent by the lack of standards both in the terminology and the methodology used to measure activity levels. This has impeded comprehensive metaanalysis. The adoption of simple objective measures of activity levels would assist in providing insight into the real impact and link between activity and AD. Given the potential clinical impact of activity in this patient population, controlling and measuring this variable should be considered.

Actigraphy is a valuable tool to objectively define patient populations in terms of their sleep and activity patterns. These devices have low patient and site burden and have the ability to generate data 24 hours a day for long periods of time in a real world, nonclinical setting, suppositionally converting daily physical activities into a digital signal. This technology has the potential to simplify and standardise the collection of objective, clinically relevant endpoints generated in real world nonclinical settings. These data used in combination with other physiological data could be used to generate new digital biomarkers that have the potential to make a real difference in AD drug development trials. Significant additional research is required to generate and validate new biomarkers, and as a first step, the adoption of a standardised approach to the collection and capture of sleep and activity data would greatly assist this process.

Conflict of interest: There are no conflicts of interest.

\section{References}

1. Morgenthaler T, Alessi C, Friedman L,. Practice Paramters for the use of Actigraphy in the Assessment of Sleep and Sleep Disorder: An update 2007. Sleep 30, (4):521-529.

2. Colley RC, Garriguet D, Janssen I et al. Physical activity of Canadian adulats: Accelerometer results from the 2007 to 2009 CHMS. Heath reports, 2011 March (22);1: 1-7

3. Mc Carthy M, Muehlhausen W, (2015). «Can Actigraphy Outcome Measures from existing clinical Trials provide a framework for sleep and activity endpoints standards in the clincal trials of the Future.ISPOR, (p. Poster PRM239). Milan.

4. Alzheimers and Dement. Alzheimers's Association Report; Alzheimers's disease facts and figures. 2013 (9)208-245.

5. Schüler P,. High AD Study Complexity Low Success Rate. The Journal of Prevention of Alzheimer's Disease, Volume 1 Number 1, 2014.

6. Ancoli-Israel S, Cole R, Alessi C et al. The role of actigraphy in the study of sleep and circadian rhythms. American Acadmey of Sleep Medicine. Sleep 
2003; 26(3): 342-92

7. 2008 Physical Activity Guidelines for Americans. (2008). Retrieved November 9th, 2015, from http:/ / health.gov/ paguidelines/guidelines

8. Singer C, Tractenberg RE, Kaye J, et al. Alzheimer's Disease Cooperative Study. A multicenter, placebo-controlled trial of melatonin for sleep disturbance in Alzheimer's disease. Sleep. 2003 Nov 1;26(7):893-901

9. Ju E, McLeland J.S., Toedebusch C.D., et al. JAMA Neurology. 2013 pp. 587593.

10. Musiek E.S, Xiong D.D, Holtzman D.M, Sleep, Circadian Rhythm and the Pathogensis of Alzheimers Disease. Experimental and Molecular Medicine, 2015, 47. pp. 1-8.

11. Da Silva RAPC. Sleep disturbances and mild cognitive impairment: a Review. Sleep Science. 2015 Jan-March (8) 36-41.

12. Spira A.P, Gamaldo A.A., An Y, et al Self-reported Sleep and $\beta$-Amyloid Deposition in Community-Dwelling Older Adults. JAMA Neurol. 2013 December $1: 70(12): 1537-1543$

13. Virta J.J, Heikkita K., Perola M, et al. Midlife Sleep Characteristics Associated with Late Life Cognitive Function. Sleep, 2013. Vol 36, No 10. 1533-1541.

14. Camagaros E.E., Louzada F.M., Nobrega O.T., Wrist actigraphy for measuring sleep in intervention studeis with Alzheimer's disease patients: Application, usefulneress and challenges. Sleep Medicine Reviews, 201317 475-488.

15. Malow B., Peterson B., A novel actigraphy analusis method for detecting the effects of treatment on disturbed sleep in children with autism. 2014 APSS, (p. Poster 1060).

16. Haack M, Scott-Sutherland J, Santangelo G, Simpson NS, Sethna N, Mullington JM. Pain sensitivity and modulation in primary insomnia,
European Journal of Pain, 2012 16:522-533.

17. Vanaparthy R, Mota P, Khan R, Ehsan M, Qureshi A, ZuWallack R, Leidy N A longitudinal assessment of sleep variables during exacerbations of chronic obstructive pulmonary disease. Chron Respir Dis. 2015 Nov;12(4):299-304

18. Lauren D, Verreault R, Lindsay J, et al. Physical Activity and Risk of Cognitive Impariment and Dementia in Elderly Persons. Arch Neurol. 2001;58:498-504

19. Rovio S, Kareholt I, Helkala E. Leisure-time physical activity at midlife and the risk of dementia and Alzheimer's disease. Lancet Neurol 2005;4:705-11.

20. Brown B, Peiffer J, Martins R, et al. Multiple effects of physical activity on molecular and cognitive signs of brain aging: can exercise slow neurodegeneration and delay Alzheimer's disease? Molecular Psychiatry, 2013; 864-874

21. Lautenschlager NT, Cox KL, Flicker L, et al Effect of physical activity oon cognitive function in older asults at risk for Alzheimer disease: a randomized trial. JAMA. 2008 Spe3:300 (9): 1027-37.

22. Nemati Karimooy N, Hosseini M, Nemati M, Esmaily HO. Lifelong physical activity affects mini-mental state exam scores in individuals over 55 years of age. J Body Mov Ther. 2012 Apr;16(2):230-235.

23. Graff-Radford N. R, (2011, April 28). «Can Aerobic Exercise Protect against Dementia?». Alzheimer's Research and Therapy, 2011.

24. Young J, Angevaren M, Rusted L et al. Aerobic exercise to improve cognitive function in older people without know cognitive impairment. Cochrane Database of Systematic Reviews 2015, Issue 4. Art. No.: CD005381. DOI: 10.1002/14651858.CD005381.pub4. 